Network Working Group

Request for Comments: 2579

STD : 58

Obsoletes: 1903

Category: Standards Track

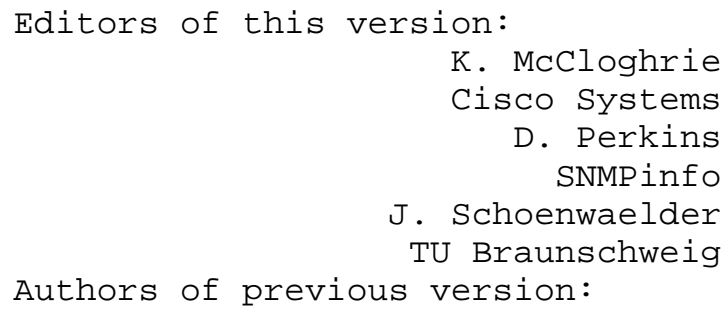

Textual Conventions for SMIv2

Status of this Memo

This document specifies an Internet standards track protocol for the Internet community, and requests discussion and suggestions for improvements. Please refer to the current edition of the "Internet Official Protocol Standards" (STD 1) for the standardization state and status of this protocol. Distribution of this memo is unlimited.

Copyright Notice

Copyright (C) The Internet Society (1999). All Rights Reserved.

Table of Contents

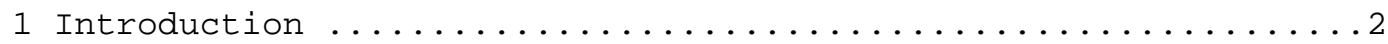

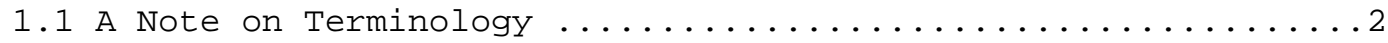

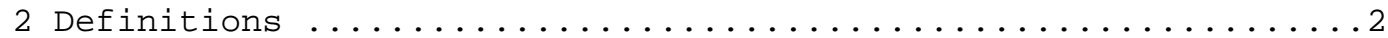

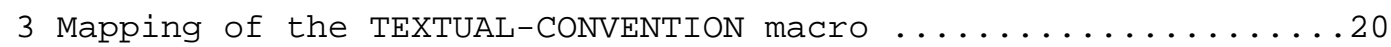

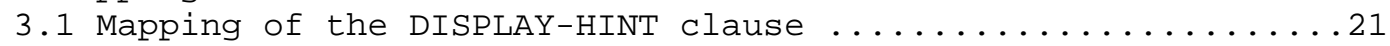

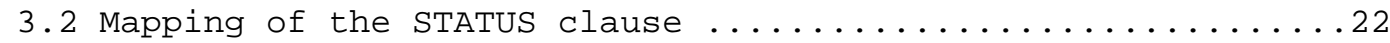

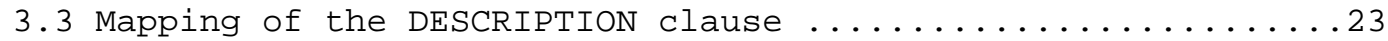

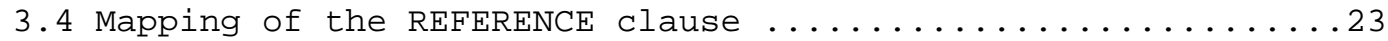

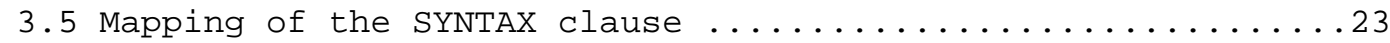

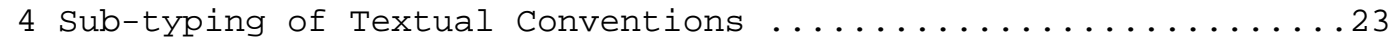

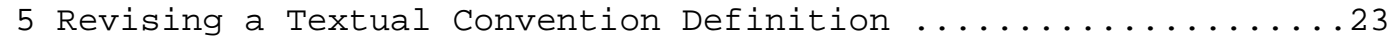

McCloghrie, et al. Standards Track 


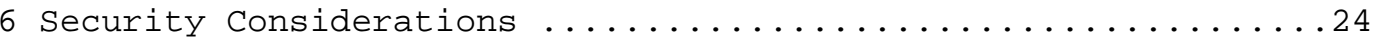

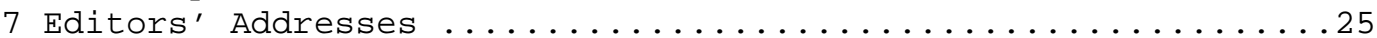

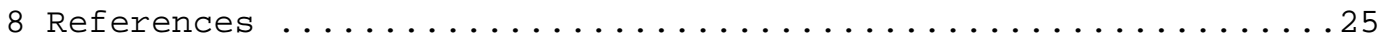

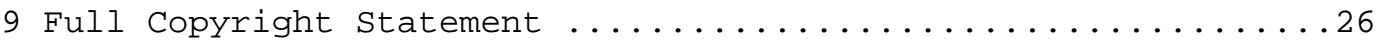

1. Introduction

Management information is viewed as a collection of managed objects, residing in a virtual information store, termed the Management Information Base (MIB). Collections of related objects are defined in MIB modules. These modules are written using an adapted subset of OSI's Abstract Syntax Notation One, ASN.1 (1988) [1], termed the Structure of Management Information (SMI) [2].

When designing a MIB module, it is often useful to define new types similar to those defined in the SMI. In comparison to a type defined in the SMI, each of these new types has a different name, a similar syntax, but a more precise semantics. These newly defined types are termed textual conventions, and are used for the convenience of humans reading the MIB module. It is the purpose of this document to define the initial set of textual conventions available to all MIB modules.

Objects defined using a textual convention are always encoded by means of the rules that define their primitive type. However, textual conventions often have special semantics associated with them. As such, an ASN.1 macro, TEXTUAL-CONVENTION, is used to concisely convey the syntax and semantics of a textual convention.

1.1. A Note on Terminology

For the purpose of exposition, the original Structure of Management Information, as described in RFCs 1155 (STD 16), 1212 (STD 16), and RFC 1215, is termed the SMI version 1 (SMIV1). The current version of the structure of Management Information is termed SMI version 2 (SMIV2).

2. Definitions

SNMPV2-TC DEFINITIONS : := BEGIN

IMPORTS

TimeTicks FROM SNMPV2-SMI;

-- definition of textual conventions

TEXTUAL-CONVENTION MACRO : := 
BEGIN

TYPE NOTATION : :=

Displaypart

"STATUS" Status

"DESCRIPTION" Text

ReferPart

"SYNTAX" Syntax

VALUE NOTATION : :=

value (VALUE Syntax) -- adapted ASN.1

Displaypart : :=

"DISPLAY-HINT" Text

| empty

Status : :=

"current"

"deprecated"

"obsolete"

Referpart : :=

"REFERENCE" Text

| empty

-- a character string as defined in [2]

Text : := value (IA5String)

Syntax ::= -- Must be one of the following:

-- a base type (or its refinement), or

-- a BITS pseudo-type

type

| "BITS" "\{" NamedBits " $\}$ "

NamedBits $::=$ NamedBit

| NamedBits "," NamedBit

NamedBit ::= identifier "(" number ")" -- number is nonnegative

END

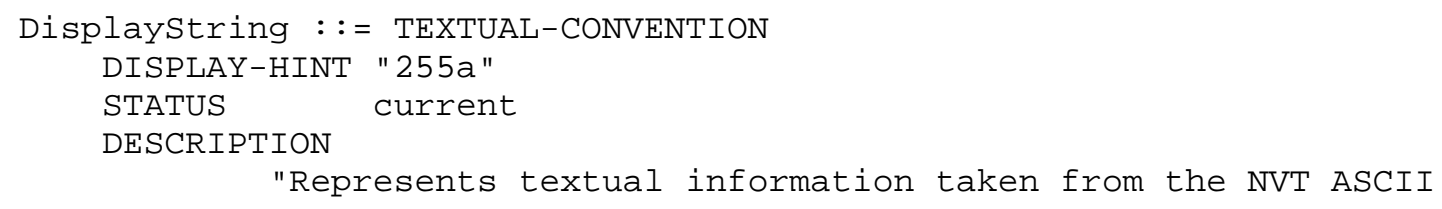

"Represents textual information taken from the NVT ASCII 


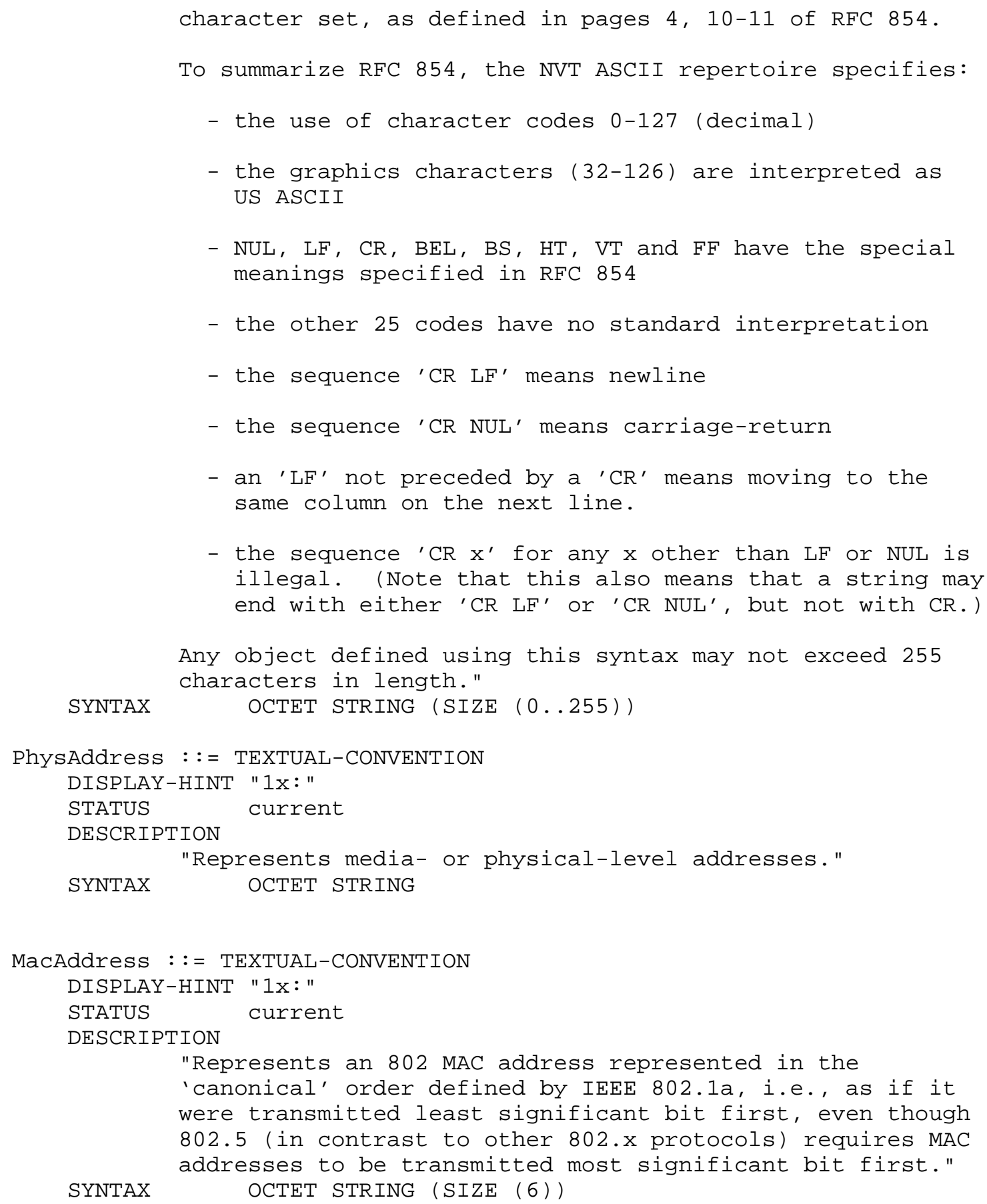




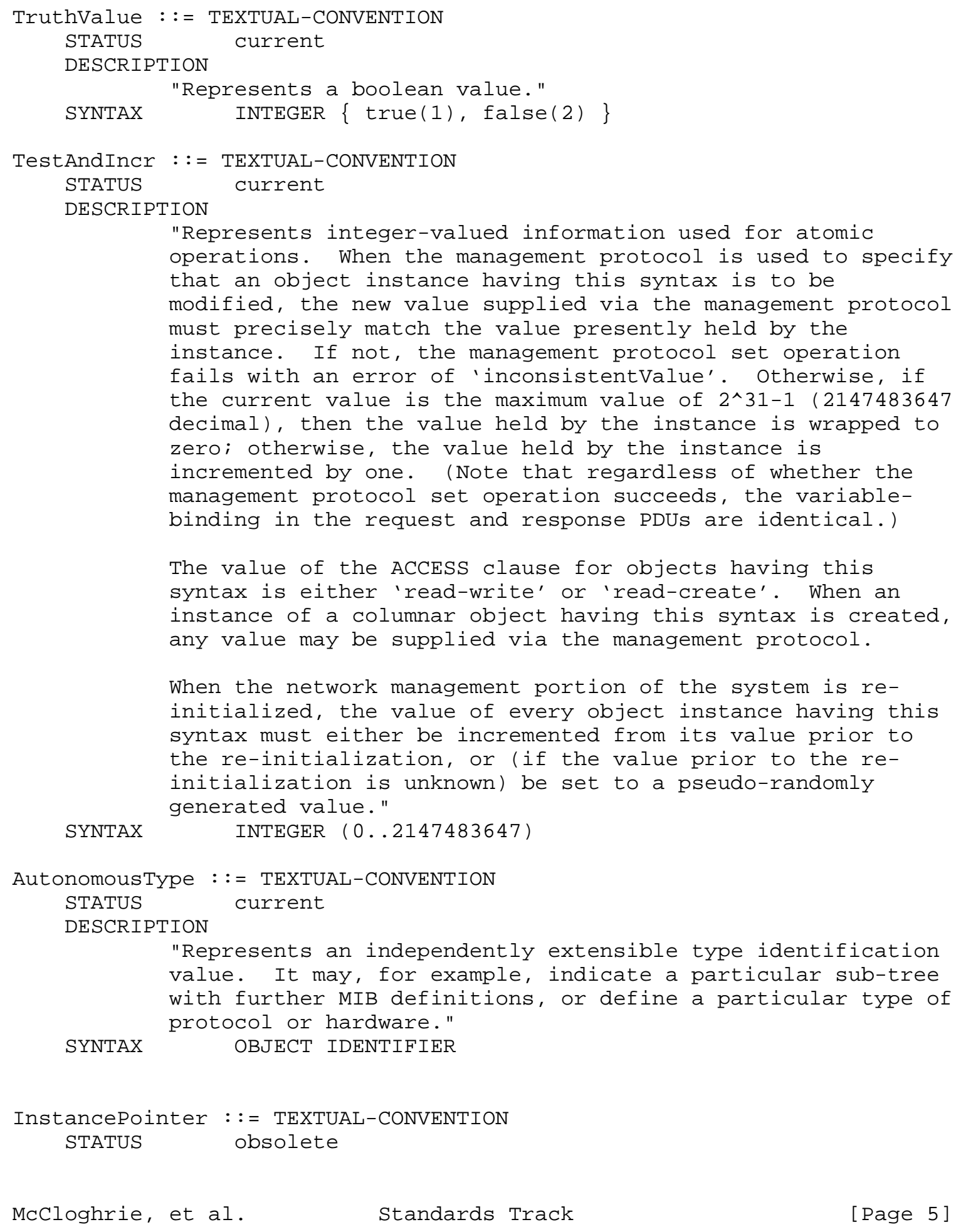




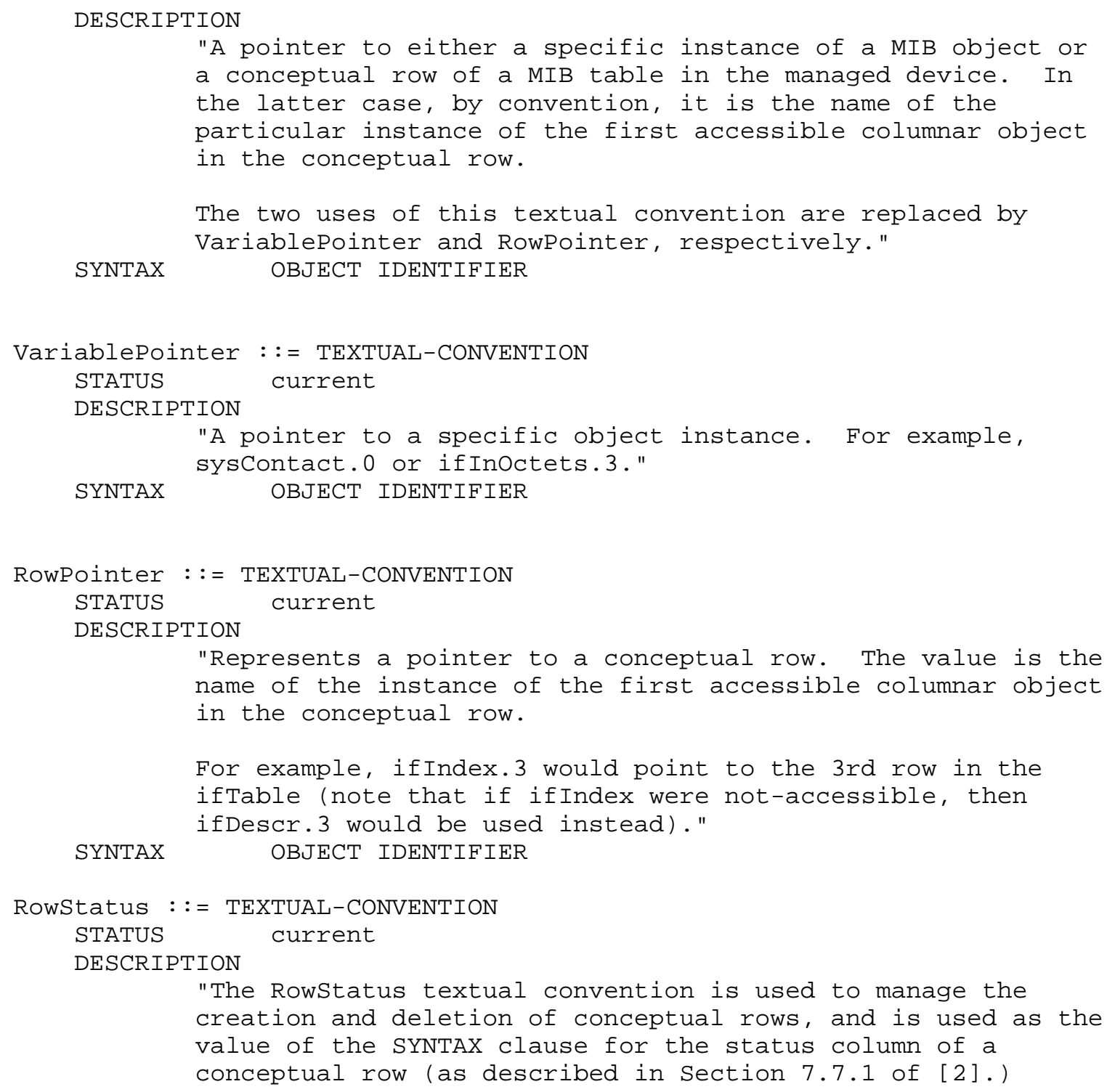


The status column has six defined values:

- 'active', which indicates that the conceptual row is available for use by the managed device;

- 'notInService', which indicates that the conceptual row exists in the agent, but is unavailable for use by the managed device (see NOTE below); 'notInService' has no implication regarding the internal consistency of the row, availability of resources, or consistency with the current state of the managed device;

- 'notReady', which indicates that the conceptual row exists in the agent, but is missing information necessary in order to be available for use by the managed device (i.e., one or more required columns in the conceptual row have not been instanciated);

- 'createAndGo', which is supplied by a management station wishing to create a new instance of a conceptual row and to have its status automatically set to active, making it available for use by the managed device;

- 'createAndWait', which is supplied by a management station wishing to create a new instance of a conceptual row (but not make it available for use by the managed device); and,

- 'destroy', which is supplied by a management station wishing to delete all of the instances associated with an existing conceptual row.

Whereas five of the six values (all except 'notReady') may be specified in a management protocol set operation, only three values will be returned in response to a management protocol retrieval operation: 'notReady', 'notInService' or 'active'. That is, when queried, an existing conceptual row has only three states: it is either available for use by the managed device (the status column has value 'active'); it is not available for use by the managed device, though the agent has sufficient information to attempt to make it so (the status column has value 'notInservice'); or, it is not available for use by the managed device, and an attempt to make it so would fail because the agent has insufficient information (the state column has value 'notReady'). 


\section{NOTE WELL}

This textual convention may be used for a MIB table, irrespective of whether the values of that table's conceptual rows are able to be modified while it is active, or whether its conceptual rows must be taken out of service in order to be modified. That is, it is the responsibility of the DESCRIPTION clause of the status column to specify whether the status column must not be 'active' in order for the value of some other column of the same conceptual row to be modified. If such a specification is made, affected columns may be changed by an SNMP set PDU if the RowStatus would not be equal to 'active' either immediately before or after processing the PDU. In other words, if the PDU also contained a varbind that would change the Rowstatus value, the column in question may be changed if the Rowstatus was not equal to 'active' as the PDU was received, or if the varbind sets the status to a value other than 'active'.

Also note that whenever any elements of a row exist, the Rowstatus column must also exist. 
To summarize the effect of having a conceptual row with a status column having a SYNTAX clause value of Rowstatus, consider the following state diagram:

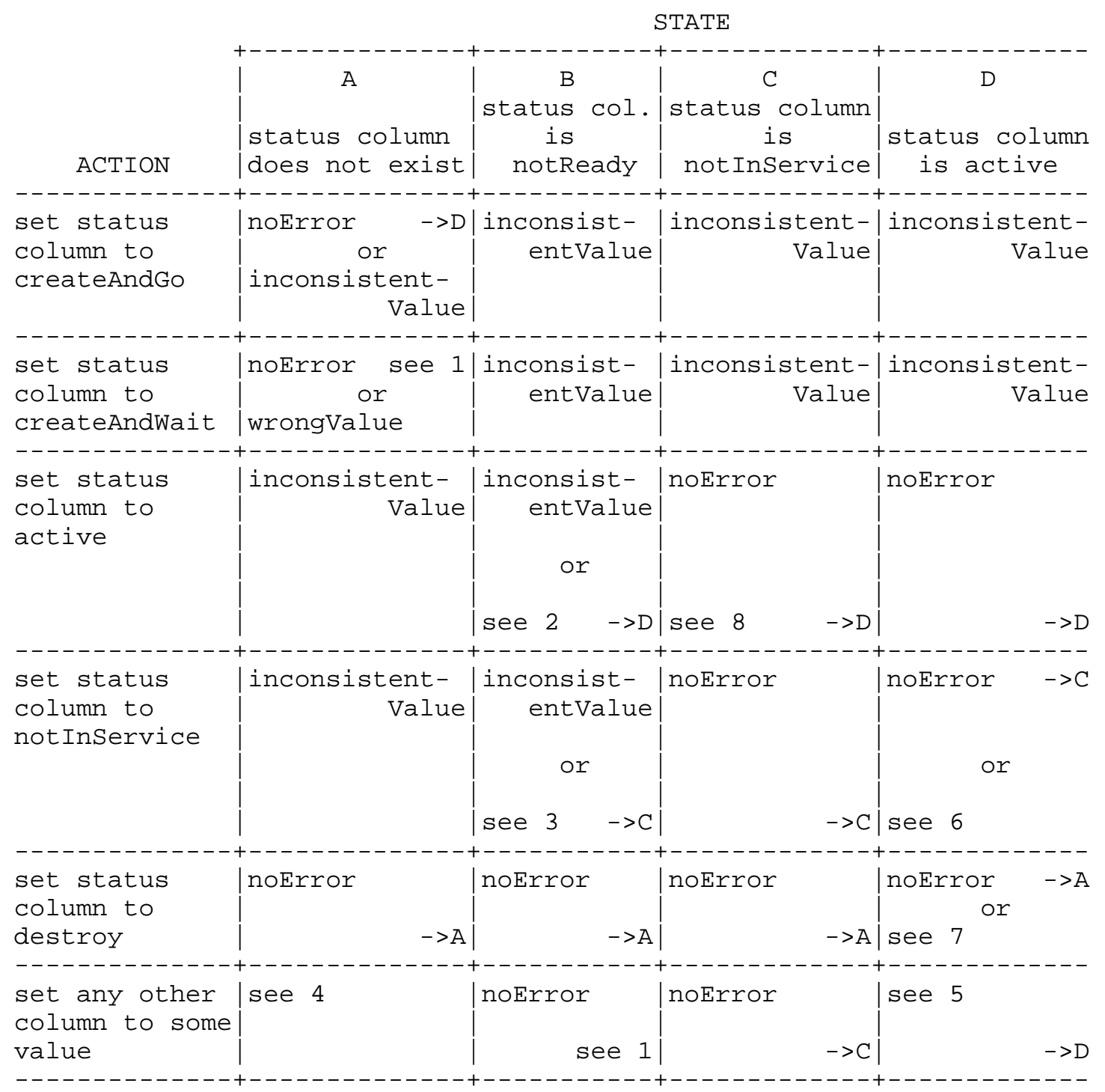

(1) goto $\mathrm{B}$ or $\mathrm{C}$, depending on information available to the agent.

(2) if other variable bindings included in the same PDU, 
provide values for all columns which are missing but required, and all columns have acceptable values, then return noError and goto $\mathrm{D}$.

(3) if other variable bindings included in the same PDU, provide legal values for all columns which are missing but required, then return noError and goto $\mathrm{C}$.

(4) at the discretion of the agent, the return value may be either:

inconsistentName: because the agent does not choose to create such an instance when the corresponding

RowStatus instance does not exist, or

inconsistentValue: if the supplied value is inconsistent with the state of some other MIB object's value, or

noError: because the agent chooses to create the instance.

If noError is returned, then the instance of the status column must also be created, and the new state is B or C, depending on the information available to the agent. If inconsistentName or inconsistentValue is returned, the row remains in state $A$.

(5) depending on the MIB definition for the column/table, either noError or inconsistentValue may be returned.

(6) the return value can indicate one of the following errors:

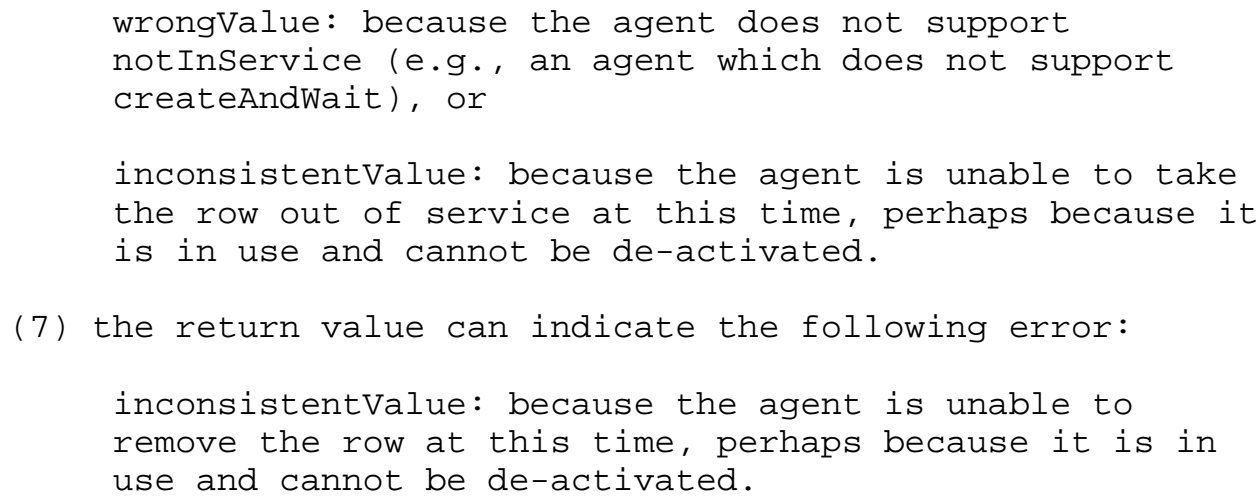


(8) the transition to D can fail, e.g., if the values of the conceptual row are inconsistent, then the error code would be inconsistentvalue.

NOTE: Other processing of (this and other varbinds of) the set request may result in a response other than noError being returned, e.g., wrongValue, nocreation, etc.

\section{Conceptual Row Creation}

There are four potential interactions when creating a conceptual row: selecting an instance-identifier which is not in use; creating the conceptual row; initializing any objects for which the agent does not supply a default; and, making the conceptual row available for use by the managed device.

Interaction 1: Selecting an Instance-Identifier

The algorithm used to select an instance-identifier varies

for each conceptual row. In some cases, the instanceidentifier is semantically significant, e.g., the destination address of a route, and a management station selects the instance-identifier according to the semantics.

In other cases, the instance-identifier is used solely to distinguish conceptual rows, and a management station without specific knowledge of the conceptual row might examine the instances present in order to determine an unused instance-identifier. (This approach may be used, but it is often highly sub-optimal; however, it is also a questionable practice for a naive management station to attempt conceptual row creation.)

Alternately, the MIB module which defines the conceptual row might provide one or more objects which provide assistance in determining an unused instance-identifier. For example, if the conceptual row is indexed by an integer-value, then an object having an integer-valued SYNTAX clause might be defined for such a purpose, allowing a management station to issue a management protocol retrieval operation. In order to avoid unnecessary collisions between competing management stations, 'adjacent' retrievals of this object should be different.

Finally, the management station could select a pseudo-random number to use as the index. In the event that this index 
was already in use and an inconsistentValue was returned in response to the management protocol set operation, the management station should simply select a new pseudo-random number and retry the operation.

A MIB designer should choose between the two latter algorithms based on the size of the table (and therefore the efficiency of each algorithm). For tables in which a large number of entries are expected, it is recommended that a MIB object be defined that returns an acceptable index for creation. For tables with small numbers of entries, it is recommended that the latter pseudo-random index mechanism be used.

Interaction 2: Creating the Conceptual Row

Once an unused instance-identifier has been selected, the management station determines if it wishes to create and activate the conceptual row in one transaction or in a negotiated set of interactions.

Interaction 2a: Creating and Activating the Conceptual Row

The management station must first determine the column requirements, i.e., it must determine those columns for which it must or must not provide values. Depending on the complexity of the table and the management station's knowledge of the agent's capabilities, this determination can be made locally by the management station. Alternately, the management station issues a management protocol get operation to examine all columns in the conceptual row that it wishes to create. In response, for each column, there are three possible outcomes:

- a value is returned, indicating that some other management station has already created this conceptual row. We return to interaction 1 .

- the exception 'noSuchInstance' is returned, indicating that the agent implements the object-type associated with this column, and that this column in at least one conceptual row would be accessible in the MIB view used by the retrieval were it to exist. For those columns to which the agent provides read-create access, the 'nosuchInstance' exception tells the management station that it should supply a value for this column when the conceptual row is to be created. 
- the exception 'noSuchobject' is returned, indicating that the agent does not implement the object-type associated with this column or that there is no conceptual row for which this column would be accessible in the MIB view used by the retrieval. As such, the management station can not issue any management protocol set operations to create an instance of this column.

Once the column requirements have been determined, a management protocol set operation is accordingly issued. This operation also sets the new instance of the status column to 'createAndGo'.

When the agent processes the set operation, it verifies that it has sufficient information to make the conceptual row available for use by the managed device. The information available to the agent is provided by two sources: the management protocol set operation which creates the conceptual row, and, implementation-specific defaults supplied by the agent (note that an agent must provide implementation-specific defaults for at least those objects which it implements as read-only). If there is sufficient information available, then the conceptual row is created, a 'noError' response is returned, the status column is set to 'active', and no further interactions are necessary (i.e., interactions 3 and 4 are skipped). If there is insufficient information, then the conceptual row is not created, and the set operation fails with an error of 'inconsistentValue'. On this error, the management station can issue a management protocol retrieval operation to determine if this was because it failed to specify a value for a required column, or, because the selected instance of the status column already existed. In the latter case, we return to interaction 1. In the former case, the management station can re-issue the set operation with the additional information, or begin interaction 2 again using 'createAndWait' in order to negotiate creation of the conceptual row. 


\author{
NOTE WELL
}

\begin{abstract}
Regardless of the method used to determine the column requirements, it is possible that the management station might deem a column necessary when, in fact, the agent will not allow that particular columnar instance to be created or written. In this case, the management protocol set operation will fail with an error such as 'nocreation' or 'notWritable'. In this case, the management station decides whether it needs to be able to set a value for that particular columnar instance. If not, the management station re-issues the management protocol set operation, but without setting a value for that particular columnar instance; otherwise, the management station aborts the row creation algorithm.
\end{abstract}

Interaction 2b: Negotiating the Creation of the Conceptual Row

The management station issues a management protocol set operation which sets the desired instance of the status column to 'createAndWait'. If the agent is unwilling to process a request of this sort, the set operation fails with an error of 'wrongValue'. (As a consequence, such an agent must be prepared to accept a single management protocol set operation, i.e., interaction 2 a above, containing all of the columns indicated by its column requirements.) Otherwise, the conceptual row is created, a 'noError' response is returned, and the status column is immediately set to either 'notInService' or 'notReady', depending on whether it has sufficient information to (attempt to) make the conceptual row available for use by the managed device. If there is sufficient information available, then the status column is set to 'notInService'; otherwise, if there is insufficient information, then the status column is set to 'notReady'. Regardless, we proceed to interaction 3 .

Interaction 3: Initializing non-defaulted objects

The management station must now determine the column requirements. It issues a management protocol get operation to examine all columns in the created conceptual row. In the response, for each column, there are three possible outcomes: 
- a value is returned, indicating that the agent implements the object-type associated with this column and had sufficient information to provide a value. For those columns to which the agent provides read-create access (and for which the agent allows their values to be changed after their creation), a value return tells the management station that it may issue additional management protocol set operations, if it desires, in order to change the value associated with this column.

- the exception 'noSuchInstance' is returned, indicating that the agent implements the object-type associated with this column, and that this column in at least one conceptual row would be accessible in the MIB view used by the retrieval were it to exist. However, the agent does not have sufficient information to provide a value, and until a value is provided, the conceptual row may not be made available for use by the managed device. For those columns to which the agent provides read-create access, the 'nosuchInstance' exception tells the management station that it must issue additional management protocol set operations, in order to provide a value associated with this column.

- the exception 'noSuchobject' is returned, indicating that the agent does not implement the object-type associated with this column or that there is no conceptual row for which this column would be accessible in the MIB view used by the retrieval. As such, the management station can not issue any management protocol set operations to create an instance of this column.

If the value associated with the status column is 'notReady', then the management station must first deal with all 'noSuchInstance' columns, if any. Having done so, the value of the status column becomes 'notInService', and we proceed to interaction 4 . 
Interaction 4: Making the Conceptual Row Available

Once the management station is satisfied with the values associated with the columns of the conceptual row, it issues a management protocol set operation to set the status column to 'active'. If the agent has sufficient information to make the conceptual row available for use by the managed device, the management protocol set operation succeeds (a 'noError' response is returned). Otherwise, the management protocol set operation fails with an error of

'inconsistentValue'.

\section{NOTE WELL}

A conceptual row having a status column with value 'notInService' or 'notReady' is unavailable to the managed device. As such, it is possible for the managed device to create its own instances during the time between the management protocol set operation which sets the status column to 'createAndWait' and the management protocol set operation which sets the status column to 'active'. In this case, when the management protocol set operation is issued to set the status column to 'active', the values held in the agent supersede those used by the managed device.

If the management station is prevented from setting the status column to 'active' (e.g., due to management station or network failure) the conceptual row will be left in the 'notInService' or 'notReady' state, consuming resources indefinitely. The agent must detect conceptual rows that have been in either state for an abnormally long period of time and remove them. It is the responsibility of the DESCRIPTION clause of the status column to indicate what an abnormally long period of time would be. This period of time should be long enough to allow for human response time (including 'think time') between the creation of the conceptual row and the setting of the status to 'active'. In the absence of such information in the DESCRIPTION clause, it is suggested that this period be approximately 5 minutes in length. This removal action applies not only to newly-created rows, but also to previously active rows which are set to, and left in, the notInService state for a prolonged period exceeding that which is considered normal for such a conceptual row. 


\section{Conceptual Row Suspension}

When a conceptual row is 'active', the management station may issue a management protocol set operation which sets the instance of the status column to 'notInService'. If the agent is unwilling to do so, the set operation fails with an error of 'wrongValue' or 'inconsistentValue'. Otherwise, the conceptual row is taken out of service, and a 'noError' response is returned. It is the responsibility of the DESCRIPTION clause of the status column to indicate under what circumstances the status column should be taken out of service (e.g., in order for the value of some other column of the same conceptual row to be modified).

\section{Conceptual Row Deletion}

For deletion of conceptual rows, a management protocol set operation is issued which sets the instance of the status column to 'destroy'. This request may be made regardless of the current value of the status column (e.g., it is possible to delete conceptual rows which are either 'notReady', 'notInService' or 'active'.) If the operation succeeds, then all instances associated with the conceptual row are immediately removed."

SYNTAX INTEGER \{ -- the following two values are states: -- these values may be read or written active (1), not InService (2),

-- the following value is a state: -- this value may be read, but not written notReady (3),

-- the following three values are -- actions: these values may be written, -- but are never read createAndGo (4), createAndWait (5), destroy (6)

\}

Timestamp : := TEXTUAL-CONVENTION

STATUS current

DESCRIPTION

"The value of the sysUpTime object at which a specific occurrence happened. The specific occurrence must be 


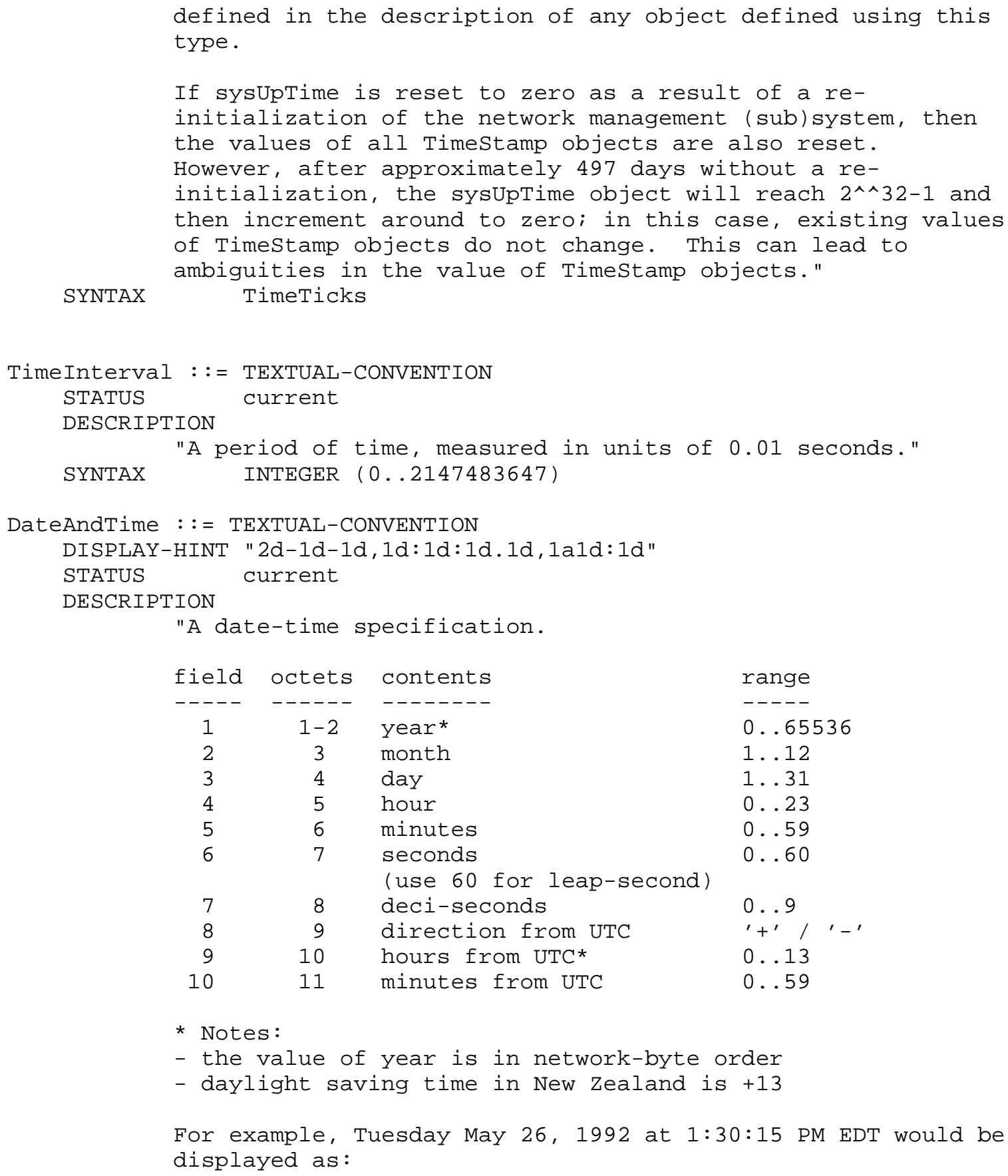




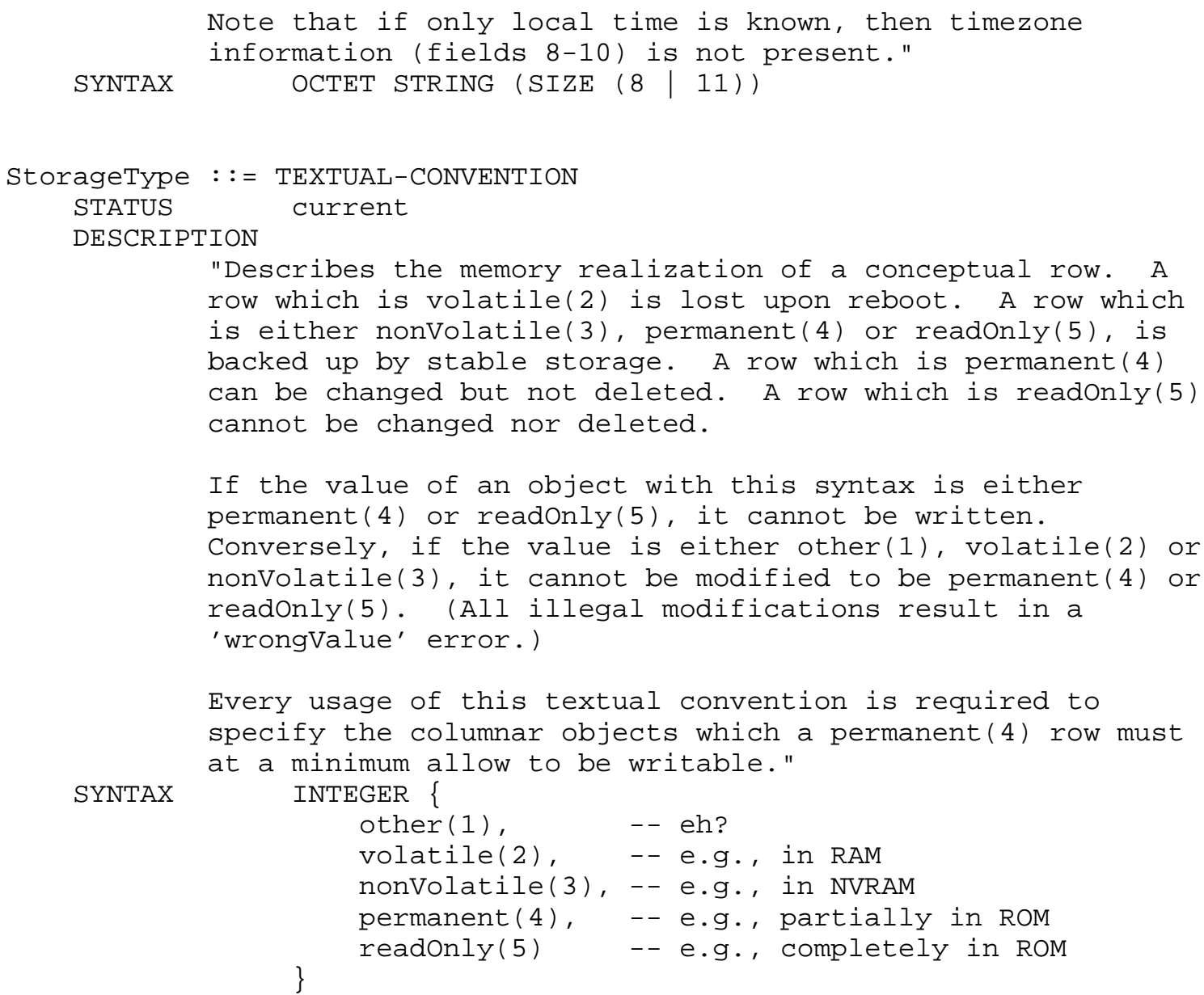

McCloghrie, et al. Standards Track [Page 19] 


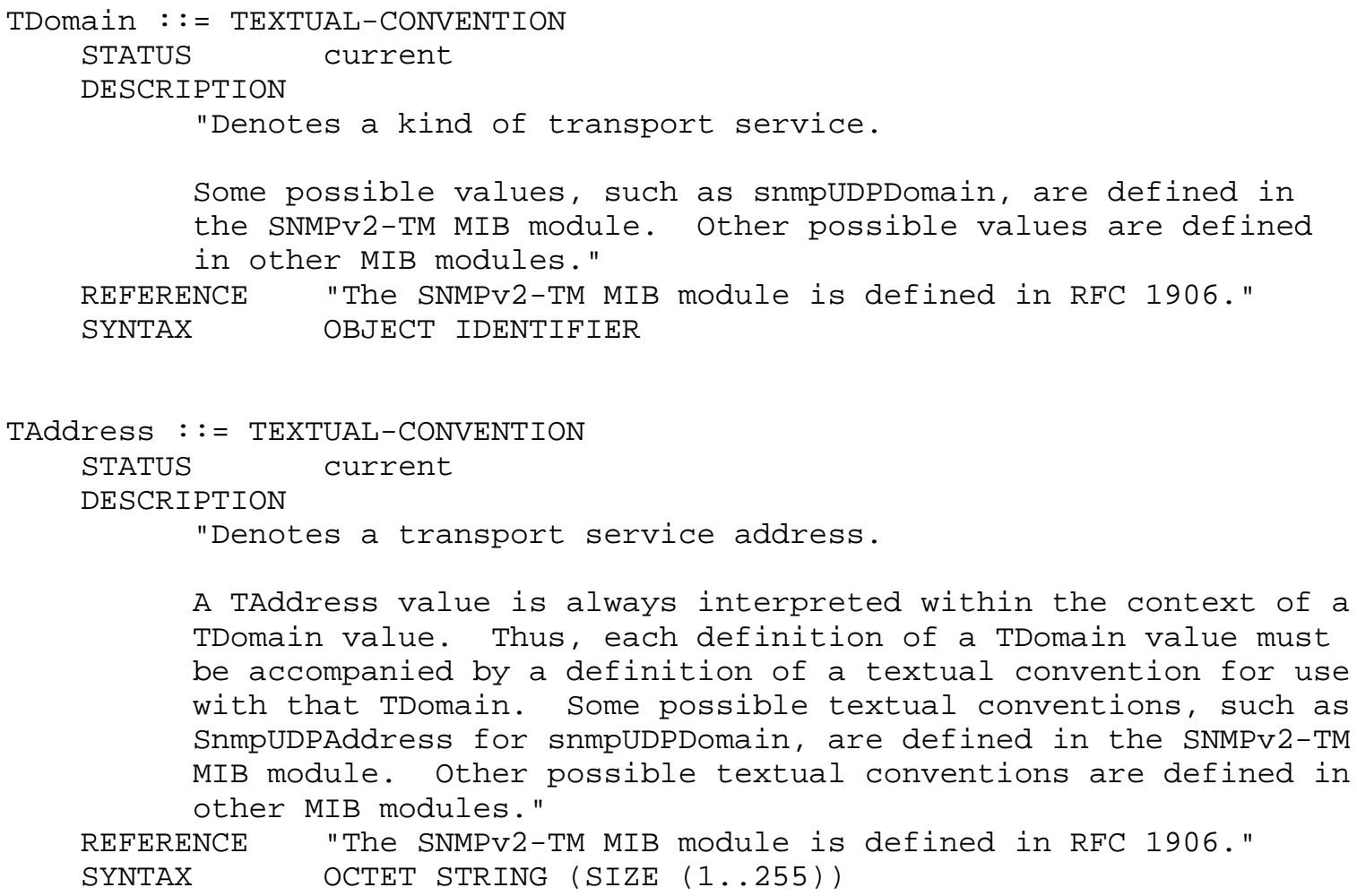

END

3. Mapping of the TEXTUAL-CONVENTION macro

The TEXTUAL-CONVENTION macro is used to convey the syntax and semantics associated with a textual convention. It should be noted that the expansion of the TEXTUAL-CONVENTION macro is something which conceptually happens during implementation and not during run-time.

The name of a textual convention must consist of one or more letters or digits, with the initial character being an upper case letter. The name must not conflict with any of the reserved words listed in section 3.7 of [2], should not consist of all upper case letters, and shall not exceed 64 characters in length. (However, names longer than 32 characters are not recommended.) The hyphen is not allowed in the name of a textual convention (except for use in information modules converted from SMIv1 which allowed hyphens in ASN.1 type assignments). Further, all names used for the textual conventions defined in all "standard" information modules shall be unique. 


\subsection{Mapping of the DISPLAY-HINT clause}

The DISPLAY-HINT clause, which need not be present, gives a hint as to how the value of an instance of an object with the syntax defined using this textual convention might be displayed. The DISPLAY-HINT clause must not be present if the Textual Convention is defined with a syntax of: OBJECT IDENTIFIER, IpAddress, Counter32, Counter64, or any enumerated syntax (BITS or INTEGER). The determination of whether it makes sense for other syntax types is dependent on the specific definition of the Textual Convention.

When the syntax has an underlying primitive type of INTEGER, the hint consists of an integer-format specification, containing two parts. The first part is a single character suggesting a display format, either: ' $x$ ' for hexadecimal, or ' $d$ ' for decimal, or ' $o$ ' for octal, or 'b' for binary. For all types, when rendering the value, leading zeros are omitted, and for negative values, a minus sign is rendered immediately before the digits. The second part is always omitted for ' $x$ ', ' $O$ ' and ' $b$ ', and need not be present for ' $d$ '. If present, the second part starts with a hyphen and is followed by a decimal number, which defines the implied decimal point when rendering the value.

For example:

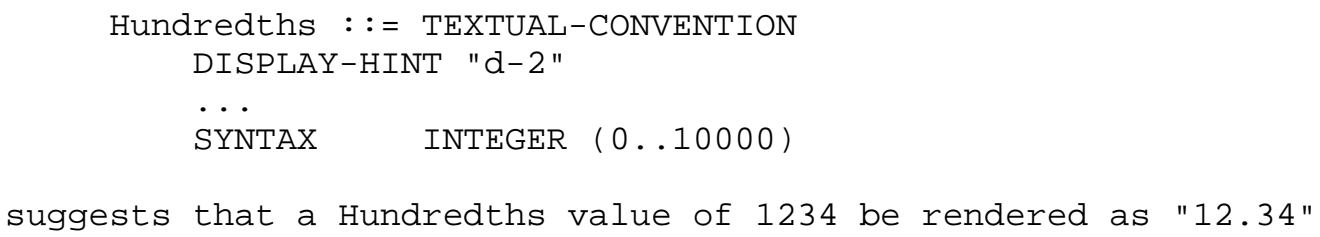

When the syntax has an underlying primitive type of OCTET STRING, the hint consists of one or more octet-format specifications. Each specification consists of five parts, with each part using and removing zero or more of the next octets from the value and producing the next zero or more characters to be displayed. The octets within the value are processed in order of significance, most significant first.

The five parts of a octet-format specification are:

(1) the (optional) repeat indicator; if present, this part is a '*', and indicates that the current octet of the value is to be used as the repeat count. The repeat count is an unsigned integer (which may be zero) which specifies how many times the remainder of this octet-format specification should be successively applied. If the repeat indicator is not present, the repeat count is one. 
(2) the octet length: one or more decimal digits specifying the number of octets of the value to be used and formatted by this octetspecification. Note that the octet length can be zero. If less than this number of octets remain in the value, then the lesser number of octets are used.

the display format, either: ' $x$ ' for hexadecimal, ' $d$ ' for decimal, 'o' for octal, 'a' for ascii, or ' $t$ ' for UTF-8. If the octet length part is greater than one, and the display format part refers to a numeric format, then network-byte ordering (big-endian encoding) is used interpreting the octets in the value. The octets processed by the ' $t$ ' display format do not necessarily form an integral number of UTF-8 characters. Trailing octets which do not form a valid UTF-8 encoded character are discarded.

(4) the (optional) display separator character; if present, this part is a single character which is produced for display after each application of this octet-specification; however, this character is not produced for display if it would be immediately followed by the display of the repeat terminator character for this octetspecification. This character can be any character other than a decimal digit and a '*'.

(5) the (optional) repeat terminator character, which can be present only if the display separator character is present and this octetspecification begins with a repeat indicator; if present, this part is a single character which is produced after all the zero or more repeated applications (as given by the repeat count) of this octet-specification. This character can be any character other than a decimal digit and a '*'.

Output of a display separator character or a repeat terminator character is suppressed if it would occur as the last character of the display.

If the octets of the value are exhausted before all the octet-format specification have been used, then the excess specifications are ignored. If additional octets remain in the value after interpreting all the octet-format specifications, then the last octet-format specification is re-interpreted to process the additional octets, until no octets remain in the value.

\subsection{Mapping of the STATUS clause}

The STATUS clause, which must be present, indicates whether this definition is current or historic.

The value "current" means that the definition is current and valid. 
The value "obsolete" means the definition is obsolete and should not be implemented and/or can be removed if previously implemented. While the value "deprecated" also indicates an obsolete definition, it permits new/continued implementation in order to foster interoperability with older/existing implementations.

3.3. Mapping of the DESCRIPTION clause

The DESCRIPTION clause, which must be present, contains a textual definition of the textual convention, which provides all semantic definitions necessary for implementation, and should embody any information which would otherwise be communicated in any ASN.1 commentary annotations associated with the object.

3.4. Mapping of the REFERENCE clause

The REFERENCE clause, which need not be present, contains a textual cross-reference to some other document, either another information module which defines a related assignment, or some other document which provides additional information relevant to this definition.

\subsection{Mapping of the SYNTAX clause}

The SYNTAX clause, which must be present, defines abstract data structure corresponding to the textual convention. The data structure must be one of the alternatives defined in the objectsyntax CHOICE or the BITS construct (see section 7.1 in [2]). Note that this means that the SYNTAX clause of a Textual Convention can not refer to a previously defined Textual Convention.

An extended subset of the full capabilities of ASN.1 (1988) subtyping is allowed, as appropriate to the underlying ASN.1 type. Any such restriction on size, range or enumerations specified in this clause represents the maximal level of support which makes "protocol sense". Restrictions on sub-typing are specified in detail in Section 9 and Appendix A of [2].

4. Sub-typing of Textual Conventions

The SYNTAX clause of a TEXTUAL CONVENTION macro may be sub-typed in the same way as the SYNTAX clause of an OBJECT-TYPE macro (see section 11 of [2]).

5. Revising a Textual Convention Definition

It may be desirable to revise the definition of a textual convention after experience is gained with it. However, changes are not allowed if they have any potential to cause interoperability problems "over 
the wire" between an implementation using an original specification and an implementation using an updated specification(s). Such changes can only be accommodated by defining a new textual convention (i.e., a new name).

The following revisions are allowed:

(1) A SYNTAX clause containing an enumerated INTEGER may have new enumerations added or existing labels changed. Similarly, named bits may be added or existing labels changed for the BITS construct.

(2) A STAtUS clause value of "current" may be revised as "deprecated" or "obsolete". Similarly, a STATUS clause value of "deprecated" may be revised as "obsolete". When making such a change, the DESCRIPTION clause should be updated to explain the rationale.

(3) A REFERENCE clause may be added or updated.

(4) A DISPLAY-HINTS clause may be added or updated.

(5) Clarifications and additional information may be included in the DESCRIPTION clause.

(6) Any editorial change.

Note that with the introduction of the TEXTUAL-CONVENTION macro, there is no longer any need to define types in the following manner:

Displaystring $::=$ OCTET STRING (SIZE $(0 . .255))$

When revising an information module containing a definition such as this, that definition should be replaced by a TEXTUAL-CONVENTION macro.

6. Security Considerations

This document defines the means to define new data types for the language used to write and read descriptions of management information. These data types have no security impact on the Internet. 
7. Editors' Addresses

Keith McCloghrie

Cisco Systems, Inc.

170 West Tasman Drive

San Jose, CA 95134-1706

USA

Phone: +14085265260

EMail: kzmecisco.com

David Perkins

SNMPinfo

3763 Benton Street

Santa Clara, CA 95051

USA

Phone: +1 408 221-8702

EMail: dperkins@snmpinfo.com

Juergen Schoenwaelder

TU Braunschweig

Bueltenweg $74 / 75$

38106 Braunschweig

Germany

Phone: +49531 391-3283

EMail: schoenwlibr.cs.tu-bs.de

\section{References}

[1] Information processing systems - Open Systems Interconnection Specification of Abstract Syntax Notation One (ASN.1), International Organization for Standardization. International Standard 8824, (December, 1987).

[2] McCloghrie, K., Perkins, D., Schoenwaelder, J., Case, J., Rose, M. and S. Waldbusser, "Structure of Management Information Version 2 (SMIv2)", STD 58, RFC 2578, April 1999.

[3] The SNMPv2 Working Group, Case, J., McCloghrie, K., Rose, M. and Waldbusser, S., "Transport Mappings for Version 2 of the" Simple Network Management Protocol (SNMPv2)", RFC 1906, January 1996. 
9. Full Copyright statement

Copyright (C) The Internet Society (1999). All Rights Reserved.

This document and translations of it may be copied and furnished to others, and derivative works that comment on or otherwise explain it or assist in its implementation may be prepared, copied, published and distributed, in whole or in part, without restriction of any kind, provided that the above copyright notice and this paragraph are included on all such copies and derivative works. However, this document itself may not be modified in any way, such as by removing the copyright notice or references to the Internet society or other Internet organizations, except as needed for the purpose of developing Internet standards in which case the procedures for copyrights defined in the Internet standards process must be followed, or as required to translate it into languages other than English.

The limited permissions granted above are perpetual and will not be revoked by the Internet society or its successors or assigns.

This document and the information contained herein is provided on an "AS IS" basis and THE INTERNET SOCIETY AND THE INTERNET ENGINEERING TASK FORCE DISCLAIMS ALL WARRANTIES, EXPRESS OR IMPLIED, INCLUDING BUT NOT LIMITED TO ANY WARRANTY THAT THE USE OF THE INFORMATION HEREIN WILL NOT INFRINGE ANY RIGHTS OR ANY IMPLIED WARRANTIES OF MERCHANTABILITY OR FITNESS FOR A PARTICULAR PURPOSE." 